bereits 2009 einen Instrumentenkasten im Internet zusammengestellt, der Anregungen für praxiserprobte und qualitätsgesicherte Maßnahmen zur Etablierung von Chancengleichheit in wissenschaftlichen Einrichtungen gibt.

Inhaltlich lassen sich einige positive Entwicklungen auf Basis der Zwischenberichte schon jetzt beobachten. So wird Gleichstellung nun fast durchgängig als Leitungsaufgabe erkannt und implementiert, vielerorts dem Thema deutlich größere strategische Bedeutung zugemessen und es wurden in den meisten Hochschulen seit der ersten Stellungnahme eine Reihe von Maßnahmen neu beschlossen und begonnen. Bis zu den Abschlussberichten 2013 hofft die DFG auf weitere Fortschritte bei der Durchsetzung von Chancengleichheit im Wissenschaftssystem.

\title{
Der „Praxistest“ beim Aufstieg im öffentlichen Dienst und Übertragungsmöglichkeit auf andere Bereiche aus Perspektive der Notariate und freien Berufe
}

\begin{abstract}
Mechtild Düsing
Mitglied des Vorstands des Deutschen Anwaltsvereins (DAV) und der Arbeitsgemeinschaft Anwältinnen sowie Vorsitzende des Genderausschusses des DAV; Rechtsanwältin und Notarin, Münster
\end{abstract}

\section{Bestandsaufnahme: Anwältinnen und Notarinnen in Deutschland}

Ein Blick in die Statistik zeigt, dass Frauen in der Anwaltschaft und speziell im Notariat (haupt- und nebenberuflich) immer noch deutlich unterrepräsentiert sind: Während die Zahl der zugelassenen Anwältinnen in Deutschland nach vierzig Jahren (1970: vier Prozent) heute 32 Prozent erreicht hat, liegt speziell die Zahl der Anwaltsnotarinnen bei nur 10,5 Prozent, die der hauptberuflichen Notarinnen bei 20,9 Prozent. Bei letzterer Gruppe lohnt ein Ost-West-Vergleich zur Erklärung des Wertes. So ist der Anteil der hauptberuflichen Notarinnen in den neuen Bundesländern aufgrund ihrer DDR-Vergangenheit deutlich höher als in den alten Ländern und beträgt heute durchschnittlich zwischen 40 und 50 Prozent. ${ }^{1}$ Im Vergleich: Die Zahl der Wirtschaftsprüferinnen in der BRD hat sich innerhalb der vergangenen fünfzehn Jahre mehr als vervierfacht, ihr Anteil im Geschlechtervergleich beträgt jedoch lediglich 14 Prozent. $^{2}$

\section{Juristinnen in Führungspositionen: Ein Blick in Gremien und Kanzleien}

Die Präsidien und Vorstände der deutschen Rechtsanwaltskammern sind unverändert männlich dominiert. Der Frauenanteil liegt hier durchschnittlich bei je 20 Prozent. Den Präsidien der Bundesnotar- und Bundesrechtsanwaltskammer gehört aktuell keine einzige Juristin an. Als positives Beispiel kann die Entwicklung im Deutschen Anwaltverein (DAV) angeführt werden. Hier gelang es, den Frauenanteil im Vorstand innerhalb von drei Jahren durch gezielte Lobbyarbeit mehr als zu verdoppeln. Für Frauen an Attraktivität zu verlieren scheinen die Großkanzleien. Ihr Anteil ist im Jahr 2009/10 um 1,2 Prozent zurückgegangen. Der Anteil der Vollpartnerinnen liegt aktuell bei 8,2 Prozent; der Anteil als Partnerinnen in Großkanzleien bei zehn Prozent. ${ }^{3}$

\section{Perspektive: Notarin und selbstständige Anwältin - ausge- wählte Strategien}

Frauen benötigen einen langen Atem - dieser Satz kann als Resümee aus der statistischen Bestandsaufnahme und zugleich als Ansporn für jede Juristin gelten. Basierend auf ihren eigenen Erfahrungen möchte die Verfasserin anderen Juristinnen ausdrücklich Mut machen, nicht zu resignieren, sondern aktiv ihre beruflichen Ziele zu verfolgen - auch über Hindernisse hinweg. Nachfolgend sollen einige ausgewählte, praxiserprobte Ansätze vorgestellt werden, die eine berufliche Perspektive als Notarin oder selbstständige Anwältin (in Führungsposition) strategisch unterstützen.

\section{„Eigene berufliche Ziele formulieren und diese konsequent verfolgen"}

Frauen sollten sich ihrer beruflichen Pläne bewusst sein und sich konkrete (Etappen-)Ziele setzen, die dann aktiv verfolgt werden. Es gilt dabei, sich von Hindernissen nicht aufhalten zu lassen, sondern diese als Chance zu begreifen, die Strategie zu optimieren (beispielsweise sich gezielte Unterstützung zu organisieren). „Und merke: Wer die Macht nicht will, erreicht sie auch nicht!"

\section{„Lobbying für sich selbst“ und „Aufbau und Pflege von Netz- werken"}

Der Eintritt in Führungsgremien gelingt für Frauen oft nicht sofort beim ersten Mal. Oft fehlt es an notwendiger Unterstützung. Nicht unterschätzt werden sollte deshalb die Möglichkeit, Lobbyarbeit für sich selbst zu betreiben. Ähnlich wie in der Politik gilt der Satz: Nur wer Mehrheiten organisieren kann, wird am Ende gewählt. Hierfür ist wichtig, sich frühzeitig Netzwerke in seinem Umfeld (in der Kanzlei oder in Gre-

\footnotetext{
1 Sämtliche Zahlen aus diesem Abschnitt basieren auf dem Datenmaterial der Bundesrechtsanwaltskammer <www.brak.de〉 (Zugriff: 12.10.2011).

2 Die Zahlen basieren auf dem Datenmaterial der Wirtschaftsprüferkammer <www.wpk.de> (Zugriff: 12.10.2011).

3 Vgl. azur (2011): 100 Top-Arbeitgeber 2011, Bocholt, S. 177-181.
} 


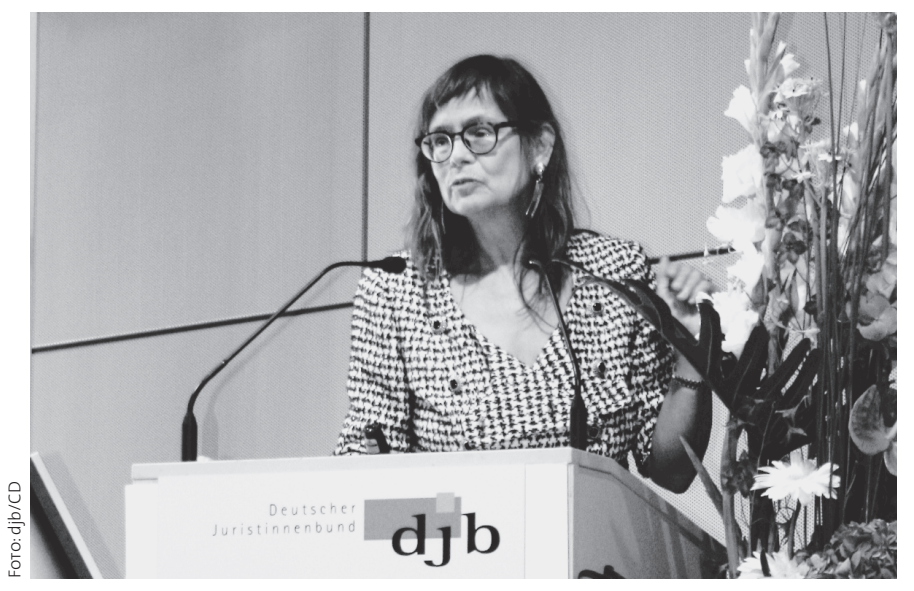

A Mechtild Düsing, Vorstandsmitglied der Arbeitsgemeinschaft Anwältinnen im DAV, Rechtsanwältin und Notarin in Münster, referiert über den Aufstieg im Öffentlichen Dienst und Übertragungsmöglichkeiten auf andere Bereiche aus der Perspektive der Notariate und freien Berufe.

mien seines Fachgebiets) aufzubauen und diese zu pflegen. Geht die Wahl dennoch verloren, so gilt an Sepp Herberger angelehnt: Nach der Wahl ist vor der Wahl!

\section{"Besetzung strategischer Ämter"}

Frauenförderung funktioniert zudem über die Besetzung strategischer Ämter in Institutionen, wie zum Beispiel das der
Genderbeauftragten oder der Vorsitzenden des Genderausschusses. Hier kann Frauenförderpolitik aktiv gestaltet werden. Beispielhaft soll hier der Genderausschuss des DAV genannt werden, dem die Verfasserin vorsteht. Dieser beschäftigt sich künftig verstärkt mit Gesetzesvorhaben, insbesondere mit der Quote für Frauen in Vorständen und Aufsichtsräten.

\section{„Veränderung beginnt in den Strukturen: ausgewählte DAV- Projekte zur Frauenförderung"}

Ohne eine Veränderung der Strukturen, etwa durch die Verankerung von gezielten Frauenförderinstrumenten, wird sich jedoch auch künftig kaum der Frauenanteil unter den Anwältinnen und Notarinnen insgesamt und speziell in den Führungspositionen nachhaltig erhöhen lassen. Der DAV hat vor diesem Hintergrund verschiedene Projekte initiiert, die sich unterschiedlichen Schwerpunkten widmen, jedoch dasselbe Ziel verfolgen: Juristinnen auf ihrem Karriereweg zu unterstützen. Hingewiesen werden soll vor diesem Hintergrund auf die Projekte „Anwältin und Mutter - wie geht das?“, „Anwältinnen in die Aufsichtsräte!", das Mentoring-Projekt der Arbeitsgemeinschaft Anwältinnen im DAV sowie die Verleihung des Anwältinnenpreises. $^{4}$

4 Nähere Informationen zu den Projekten des DAV bietet die Website <www.dav.de> (Zugriff 25.10.2011).

\section{Der „Praxistest“ beim Zugang zum und Aufstieg im öffentlichen Dienst und Überlegungen für Unternehmen privater Rechtsform}

\author{
Ursula Matthiessen-Kreuder \\ Personalleiterin, Wiesbaden
}

\section{Einstieg}

Juristinnen haben in der Bundesrepublik der Nachkriegszeit wesentliche Erfolge erzielt bei der Frage, wie sich eine gleichberechtigte Teilhabe von Frauen durchsetzen lassen könnte. Ihre Instrumente waren Gesetzesvorlagen, Gesetzesauslegungen und wissenschaftliche Untersuchungen. Im djb haben sich diese Juristinnen organisiert und ihr Status als Beratungsinstanz und Nichtregierungsorganisation (NGO) steht für die Anerkennung, die sie und ihre Arbeit seit Langem genießen.

Mit der Kampagne „Mehr Frauen in Vorstände und Aufsichtsräte“ hat der djb seit dem Jahr 2009 ein wichtiges Thema aufgegriffen. Es liegt in seiner Kernkompetenz, Regelungen wie den Corporate Governance Kodex aufzugreifen, zu analysieren, frauenspezifische Interessen zu artikulieren und Änderungen und Ergänzungen vorzuschlagen.

Auf dem diesjährigen Kongress hat sich der djb mit der Frage befasst, welche tatsächlichen Folgen die frauenpoliti- schen Bemühungen der letzten Jahre insbesondere im öffentlichen Dienst für den Berufseinstieg gehabt haben.

Versucht man, die tatsächlichen Folgen aus dem Blickwinkel einer Juristin aus einem Unternehmen privater Rechtsform $\mathrm{zu}$ verstehen und zu bewerten, ergeben sich bei der Ausgangslage Gemeinsamkeiten und Unterschiede zum öffentlichen Dienst. Ein gutes juristisches Examen, das Frauen häufiger erreichen als ihre männlichen Kommilitonen, öffnet in angemessenem Verhältnis zu ihren Bewerbungen den Einstieg in den öffentlichen Dienst. Richterinnen und Staatsanwältinnen machen sich auf den Weg und bevölkern die unteren Flure der angesehenen Institutionen. Frauen mit anderen Wünschen wenden sich dem Anwaltsberuf zu und dort stellen sie in der Zwischenzeit eine große Zahl der Kanzleiinhaberinnen. Frauen sind also da.

Juristinnen sind auch da, wenn es in den Unternehmen privater Rechtsform um Einstiegsjobs in der Personalverwaltung geht. Ein Blick auf die Zahlen zeigt, dass Personalverwaltung heute fast ganz weiblich ist, jedenfalls, wenn man die Chefs nicht mitzählt. In der Personalentwicklung sind sie seltener zu 